\title{
Dextran 500 Improves Recovery of Inflammatory Markers: An In Vitro Microdialysis Study
}

\author{
Susan Giorgi-Coll, ${ }^{1, *}$ Eric Peter Thelin, ${ }^{1-3,{ }^{*}}$ Caroline Lindblad, ${ }^{2}$ Tamara Tajsic, ${ }^{1}$ Keri L.H. Carpenter, ${ }^{1,4}$ \\ Peter J.A. Hutchinson, ${ }^{1,4}$ and Adel Helmy ${ }^{1}$
}

\begin{abstract}
Cerebral microdialysis (CMD) is used in severe traumatic brain injury (TBI) in order to recover metabolites in brain extracellular fluid (ECF). To recover larger proteins and avoid fluid loss, albumin supplemented perfusion fluid (PF) has been utilized, but because of regulatory changes in the European Union, this is no longer practicable. The aim with this study was to see whether fluid, absolute (AR), and relative (RR) recovery for the novel carrier, Dextran 500, was better than conventional PF for a range of cytokines and chemokines. An in vitro setup mimicking conditions observed in the neurocritical care of TBI patients was used, utilizing 100-kDa molecular-weight cut-off CMD catheters inserted through a triple-lumen bolt cranial access device into an external solution with diluted cytokine standards in known concentrations for $48 \mathrm{~h}$ (divided into 6-h epochs). Samples were run on a 39-plex Luminex (Luminex Corporation, Austin, TX) assay to assess cytokine concentrations. We found that fluid recovery was inadequate in 50\% of epochs with conventional PF, whereas Dextran PF overcame this limitation. The AR was higher in the Dextran PF samples for a majority of cytokines, and RR was significantly increased for macrophage colony-stimulating factor and transforming growth factor-alpha. In summary, Dextran PF improved fluid and cytokine recovery as compared to conventional PF and is a suitable alternative to albumin supplemented PF for protein microdialysis.
\end{abstract}

Keywords: chemokines; cytokines; Dextran 500; in vitro; microdialysis; recovery

\section{Introduction}

C EREBRAL MICRODIALYSIS (CMD) is a technique enabling sampling from the extracellular fluid (ECF) in vivo, providing a unique opportunity to study underlying metabolic and inflammatory processes that occur in traumatic brain injury (TBI). ${ }^{1,2}$ Microdialysis sampling is based on the free diffusion of analytes across a semipermeable membrane with a nominal molecularweight cutoff (MWCO). The membrane is attached to inlet and outlet tubing through which perfusion fluid (PF) is slowly pumped and collected. ${ }^{3}$ To measure metabolites in clinical practice, such as glucose, lactate, and pyruvate, a 20-kDa MWCO is adequate and an isotonic solution, mimicking cerebrospinal fluid, is used as a carrier. ${ }^{4}$ Microdialysis of proteins is limited by both lower absolute concentrations within the brain ECF, as well as the larger molecular weight, necessitating the use of larger MWCO membranes. This causes a number of problems, including non-specific adsorption to the device materials, clogging of membranes, and protein-protein interactions, which all negatively affect recovery. ${ }^{5-7}$ A further issue with increased MWCO catheters (e.g., $100 \mathrm{kDa}$ ) is loss by convection of fluid within the catheter. ${ }^{8}$ This is attributed to the hydrostatic pressure differences (with a relatively low osmotic pressure in the PF) and referred to as ultrafiltration. This may impact on both the ability to carry out analysis on the diminished volume of fluid recovered by the catheter as well as potentially impacting on the biology of the extracellular space, such that it is not representative of the underlying processes of interest. In order to mitigate this phenomenon, addition of colloid to the PF to increase the oncotic pressure has been recommended, typically albumin. ${ }^{6,9}$ However, a regulatory reclassification of albumin within

\footnotetext{
${ }^{1}$ Division of Neurosurgery, Department of Clinical Neurosciences and ${ }^{4}$ Wolfson Brain Imaging Centre, Department of Clinical Neurosciences, University of Cambridge, Cambridge, United Kingdom.

${ }^{2}$ Department of Clinical Neuroscience, Karolinska Institutet, Stockholm, Sweden.

${ }^{3}$ Theme Neuro, Karolinska University Hospital, Stockholm, Sweden.

*These are first co-authors.

(C) Susan Giorgi-Coll et al., 2019; Published by Mary Ann Liebert, Inc. This Open Access article is distributed under the terms of the Creative Commons License (http://creativecommons.org/licenses/by/4.0), which permits unrestricted use, distribution, and reproduction in any medium, provided the original work is properly credited.
} 
the European Union as a blood product has made formulation of albumin-supplemented fluid logistically and financially impractical. Further, the theoretical risk of albumin leak and accumulation in the surrounding tissues has been raised, with potential negative consequences. ${ }^{8}$ Our group and others have shown that cytokines and chemokines are key mediators in the inflammatory processes after TBI, ${ }^{10-12}$ and in order to advance the study of brain protein recovery and potential therapeutic advances, an accurate estimation of relative recovery is necessary. Thus, it is imperative for the continued clinical use of microdialysis that alternative strategies for improving cytokine recovery which do not require blood products are developed and tested.

An alternative colloid to increase the osmotic and hydrostatic pressure of microdialysis PF is Dextran. ${ }^{13-15}$ Dextrans are branched glycans of varying molecular sizes (3-2000 kDa), of which ranges between 60 and $500 \mathrm{kDa}$ have been extensively studied in the microdialysis setting. ${ }^{7,9,16,17}$ In comparison to normal PF, and even albumin PF, studies have shown an improved recovery of macromolecules using different molecular weights and concentrations of Dextrans. ${ }^{7,9,16}$ Recent in vitro studies have suggested that a $3 \%$ Dextran 500-kDa solution is the most suitable additive given that it is large enough not to pass through the microdialysis membrane, ${ }^{18}$ maintains the greatest fluid recovery, ${ }^{7,14}$ and does not lead to an inflammatory response in the surrounding tissue. ${ }^{8}$

Thus, the aim of this study was to determine whether PF supplemented with the recently commercially available 3\% Dextran $500 \mathrm{kDa}$ (Perfusion Fluid central nervous system [CNS] Dextran; M Dialysis, Stockholm, Sweden) could improve the fluid, absolute (AR), and relative recovery (RR) of inflammatory markers (39 cytokines and chemokines) during microdialysis sampling in vitro, in comparison to normal PF available for clinical use (Perfusion Fluid CNS; M Dialysis). The two types of PF were tested using an in vitro setup that closely approximates the clinical environment, to ascertain whether Dextran would be worthwhile to use during microdialysis sampling in human patients.

\section{Methods}

\section{Materials}

All high-purity deionized water $\left(\mathrm{dH}_{2} \mathrm{O}\right)$ used was of highperformance liquid chromatography grade $(18.2 \mathrm{M} \Omega . \mathrm{cm}$, Millipore Direct Q5 UV water purification system with LC-Pak polisher; Millipore, Burlington, MA). All reagents were also of analytical grade, purchased from Sigma-Aldrich (Poole, UK), and used as received, unless otherwise stated. Sodium chloride and potassium chloride were purchased from BDH Laboratory Supplies (Poole, UK).

M Dialysis 71 CMD catheters (100-kDa nominal MWCO, polyarylethersulfone $10-\mathrm{mm}$ membrane length), microdialysis vials, Perfusion Fluid CNS, Perfusion Fluid CNS Dextran, M Dialysis 106 microdialysis pumps, and corresponding batteries and syringes were purchased from M Dialysis (Stockholm, Sweden). Both PFs contain $147 \mathrm{mM}$ of $\mathrm{NaCl}, 2.7 \mathrm{mM}$ of $\mathrm{KCl}, 1.2 \mathrm{mM}$ of $\mathrm{CaCl}_{2}$, and $0.85 \mathrm{mM}$ of $\mathrm{MgCl}_{2}$, but with an additional 3\% 500-kDa molecularweight Dextran in the Perfusion Fluid CNS Dextran. This newly commercially available product was purchased from M Dialysis.

In vitro microdialysis sampling experiments were performed using a VWR (Radnor, PA) advanced hotplate magnetic stirrer with temperature probe. Catheters were held in place during in vitro sampling using a triple-lumen cranial access device (Technicam, Newton Abbott, UK). Custom Invitrogen eBioscience ProcartaPlex $^{\mathrm{TM}}$ human cytokine and chemokine 39-plex bead assays and human cytokine and chemokine standards (referred to by the

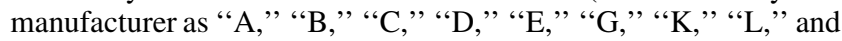
"MMP" standard mixes, plus individual standards for Galectin-3, metalloproteinase domain-containing protein [MDC], and transforming growth factor [TGF]-alpha) were purchased from Thermo Fisher (Paisley, UK). A complete list of the cytokines and chemokines analyzed is provided in Supplementary Table S1. ProcartaPlex multiplex assays were analyzed using a Luminex 200 analyzer (Luminex Corporation, Austin, TX) operating with Luminex xPONENT ${ }^{\circledR}$ software. Wash steps were performed using a ProcartaPlex hand-held magnetic plate holder.

\section{In vitro microdialysis sampling}

In vitro microdialysis sampling was performed using an artificial external solution (ES) representative of the brain extracellular environment. The ES comprised PF with $0.05 \%$ (w/v) sodium azide, $1 \mathrm{mg} / \mathrm{mL}$ of human serum albumin (HSA), and 39 human cytokines and chemokines, prepared in a 50-mL centrifuge tube $\left(\right.$ Falcon $\left.{ }^{\circledR}\right)$ as follows. Microdialysis $\mathrm{PF}$ for the external solution was made inhouse $\left(147 \mathrm{mM}\right.$ of $\mathrm{NaCl}, 2.7 \mathrm{mM}$ of $\mathrm{KCl}, 1.2 \mathrm{mM}$ of $\mathrm{CaCl}_{2}$, and $0.85 \mathrm{mM}$ of $\mathrm{MgCl}_{2} ; \mathrm{pH} \sim 6.0$ ), to the same specifications as Perfusion Fluid CNS used for CMD in patients. The mixed cytokine and chemokine standards (A, B, C, D, E, G, K, L, and MMP standard mixes, plus individual standards for Galectin-3, MDC, and TGF-alpha), received as lyophilized powders, were resuspended in accord with the manufacturers' instructions and subsequently diluted to $1: 100$ in PF with $0.05 \%$ (w/v) sodium azide and $1 \mathrm{mg} / \mathrm{mL}$ of HSA (final concentration). The total volume of the ES was $25 \mathrm{~mL}$. The final cytokine and chemokine concentrations are shown in Supplementary Table S1.

The centrifuge tube $(50 \mathrm{~mL})$ containing the ES $(25 \mathrm{~mL})$ was suspended using a clamp stand in a thermostatically controlled glycerol bath (to avoid condensation) set to $37^{\circ} \mathrm{C}$. Very gentle agitation of the external solution was applied using a magnetic stirrer. Two M Dialysis 71 brain microdialysis catheters were placed into the external solution through a triple bolt cranial access device, which was secured within the centrifuge tube using selfadhesive plastic film. Each catheter was perfused at $0.3 \mu \mathrm{L} / \mathrm{min}$ using M Dialysis 107 pumps, with syringes loaded with approximately $1.5 \mathrm{~mL}$ of either normal PF or PF containing 3\% Dextran 500. Both the normal PF and Dextran PF were used as received from the manufacturer. The microdialysate samples were collected in microdialysis vials at the end of each catheter. Pumps and collection vials were kept at the same height either side of the glycerol bath, to nullify any hydrostatic pressure differences.

Sampling was performed for $48 \mathrm{~h}$ in total; the microdialysis vials were changed every $6 \mathrm{~h}$, and sample from the ES was drawn at the $0-, 24-$, and 48 -h time points. A schematic of the in vitro sampling setup is shown in Figure 1. If no fluid was apparent in the microdialysis vial during the first 30-60 min after an exchange, that pump was flushed. This flush sequence was discarded and fluid collection started after the flush was completed. The pumps were randomly changed between fluid carriers and experiments as to not introduce any systematic bias. The in vitro sampling test was repeated in three independent experiments over the course of 4 weeks (eight time epochs per experiments, a total of 24 epochs). All samples were stored at $-80^{\circ} \mathrm{C}$ before analysis.

\section{Sample analysis}

Quantitative analysis of cytokine and chemokines was performed using custom ProcartaPlex ${ }^{\mathrm{TM}}$ human 39-plex bead-based immunoassay kits. Samples were thawed and gently mixed before analysing. In total, $25 \mu \mathrm{L}$ of sample was used per well; all samples were analyzed in duplicate. The assay was performed as per the manufacturers' instructions. Wash steps were carried out using a hand-held magnetic plate holder (ProcartaPlex). All assays were analyzed on a Luminex 200 platform. We established that Dextran 500 did not interfere with the analysis by running a standard curve using the Dextran PF as a diluent and compared it to a normal PF 


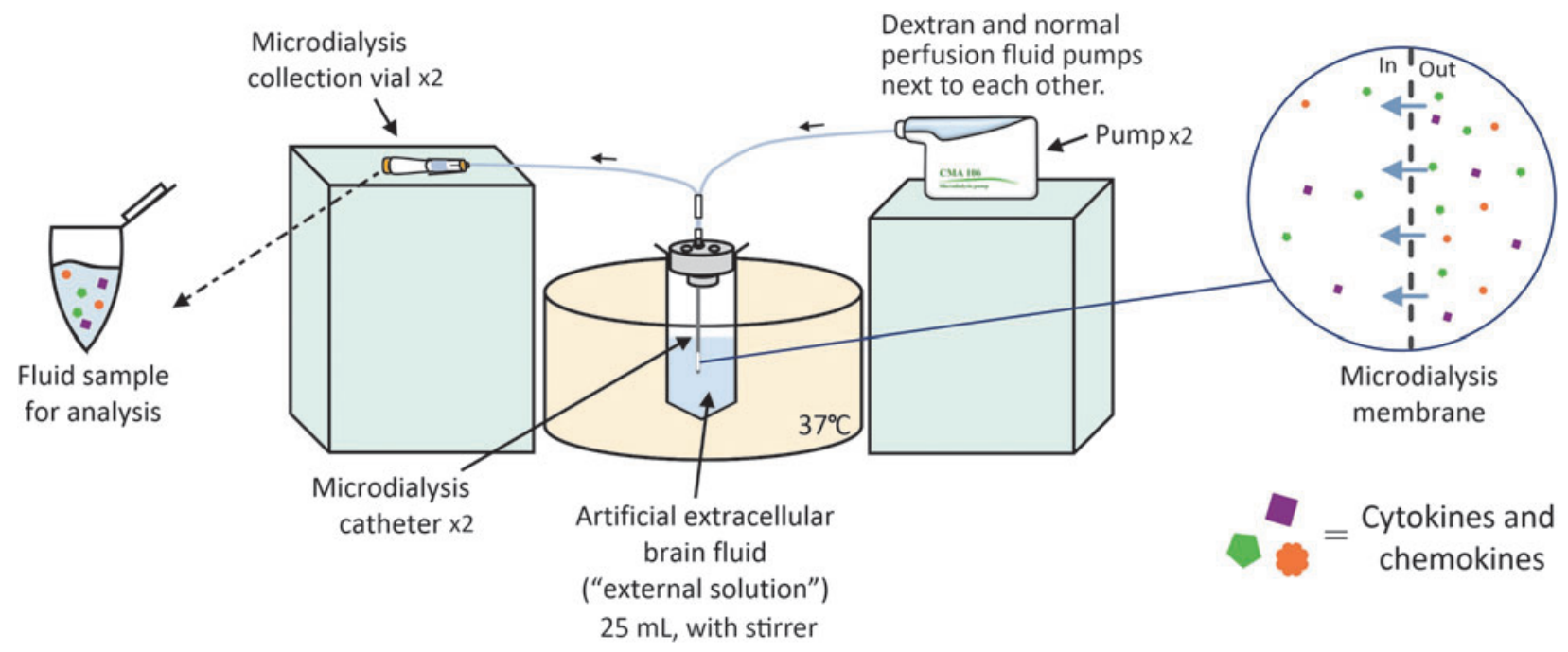

FIG. 1. Schematic overview of the experimental setup. Color image is available online.

standard curve, and Dextran PF had no discernible effect on the standard response.

\section{Statistical analysis}

All statistical analyses were conducted using the statistical software R, and its graphical interface Rstudio ${ }^{\circledR}$ (R Foundation for Statistical Computing, Vienna, Austria). ${ }^{19}$ For each described analysis below, complete case analyses were conducted. A $p$ value $\leq 0.05$ was considered significant in all analyses. Graphical presentation was conducted using the $\mathrm{R}$ packages tidyverse, ${ }^{20}$ cowplot, ${ }^{21}$ and RColorBrewer, ${ }^{22}$ unless otherwise stated.

To assess how flushing the pumps affected cytokine recovery in the remaining fluid collected in that epoch, we used the $\mathrm{R}$ package nlme ${ }^{23}$ and conducted a linear mixed model per cytokine, using cytokine recovery as a dependent variable and time together with flush as independent variables. ${ }^{24}$ In each model, the independent experiment was considered to be the random intercept. Some cytokines (Fractalkine, interferon [IFN]- $\alpha$, IFN- $\gamma$, and tumor necrosis factor [TNF] receptor type I [TNF-RI]) could not be quantified in the microdialysates, given that the concentrations recovered were below the lower limit of detection (as specified by the kit manufacturer) for the assay. Assumptions were examined graphically with regard to equal variance, linearity, and normal distribution.

Similarly, for absolute recovery analysis, time and carrier were used as independent variables in a mixed model. ${ }^{23-26}$ The dependent variable was the recovered cytokine value. For random intercepts, we used independent experiments. IFN- $\gamma$ was excluded from analysis because the returned levels were below lowest levels of detection for both PFs. Assumptions were examined graphically as described above. ${ }^{27,28}$

$\mathrm{RR}$ was calculated as the ratio between the recovered cytokine in the microdialysis vial (numerator) and the recovered cytokine in the ES (denominator) obtained concomitantly at 24 and $48 \mathrm{~h}$. For inferential analysis, cytokines exhibiting less than three positive observations (chemokine [C-C motif] ligand 20 [CCL20]/macrophage inflammatory protein 3 alpha $[\mathrm{MIP}-3 \alpha]$, Fractalkine, Galectin- 3 , IFN- $\alpha$, IFN- $\gamma$, matrix metalloproteinase [MMP]-2, TNF-RI, and vascular endothelial growth factor [VEGF]-D) were excluded. For the remainders, the cytokine retrieval capacity of the different carriers (Dextran and the conventional CNS PF) were compared using a two-sided Student's $t$-test (not assuming equal variances) or (if not normally distributed) a two-sided MannWhitney U test.

\section{Results}

\section{Fluid recovery}

Only vials perfused with the standard PF demonstrated inadequate fluid recovery in certain epochs over the three experiments and hence needed intermittent flushing in the initial phase of the epoch (Fig. 2). In total, 50\% of the vials (12 epochs) needed flushing (Fig. 2A). In these cases, sampling was briefly halted while the flush sequence was completed and resumed immediately afterward. The eluent from the flush sequence was collected separately from the sample fluid and discarded in order to avoid diluting the samples with excess fluid. For most cytokines, this procedure did not alter the absolute recovery (Table 1), but for some, notably IL-6, regulated on activation, normal T cell expressed and secreted (RANTES), and TNF, the necessary flushing sequences resulted in a significantly lower recovery in the fluid collected during the remaining of the epoch in the conventional CNS PF (Fig. 2A).

\section{Absolute recovery}

Throughout the analyzed cytokines, the absolute recovery was systematically higher for the Dextran CNS PF carrier compared to the standard CNS PF (Table 1). Two examples are highlighted in Figure 3. The samples using Dextran as carrier also had more robust results overall, as visualized by Supplementary Figure S1. Further, many protein concentrations varied over time, usually with a decreasing trajectory, presumably representing a spontaneous gradual decline of some of the proteins in the study (Table 1), presumably attributed to processes such as decomposition, aggregation, and/or adhesion to surfaces, etc.

\section{Relative recovery}

At 24 and $48 \mathrm{~h}$, the ES was sampled to enable calculation of the $\mathrm{RR}$, generating a maximum of six RRs per cytokine (two per individual experiment). Overall, there was a general trend toward a higher RR for the Dextran perfusion fluid as compared to the standard CNS (Table 1; Fig. 4). Two cytokines exhibited a significant increase in RR with Dextran PF, including M-CSF and TGF-alpha, whereas none of the cytokines' RR values were significantly higher in the standard CNS perfusion fluid. 


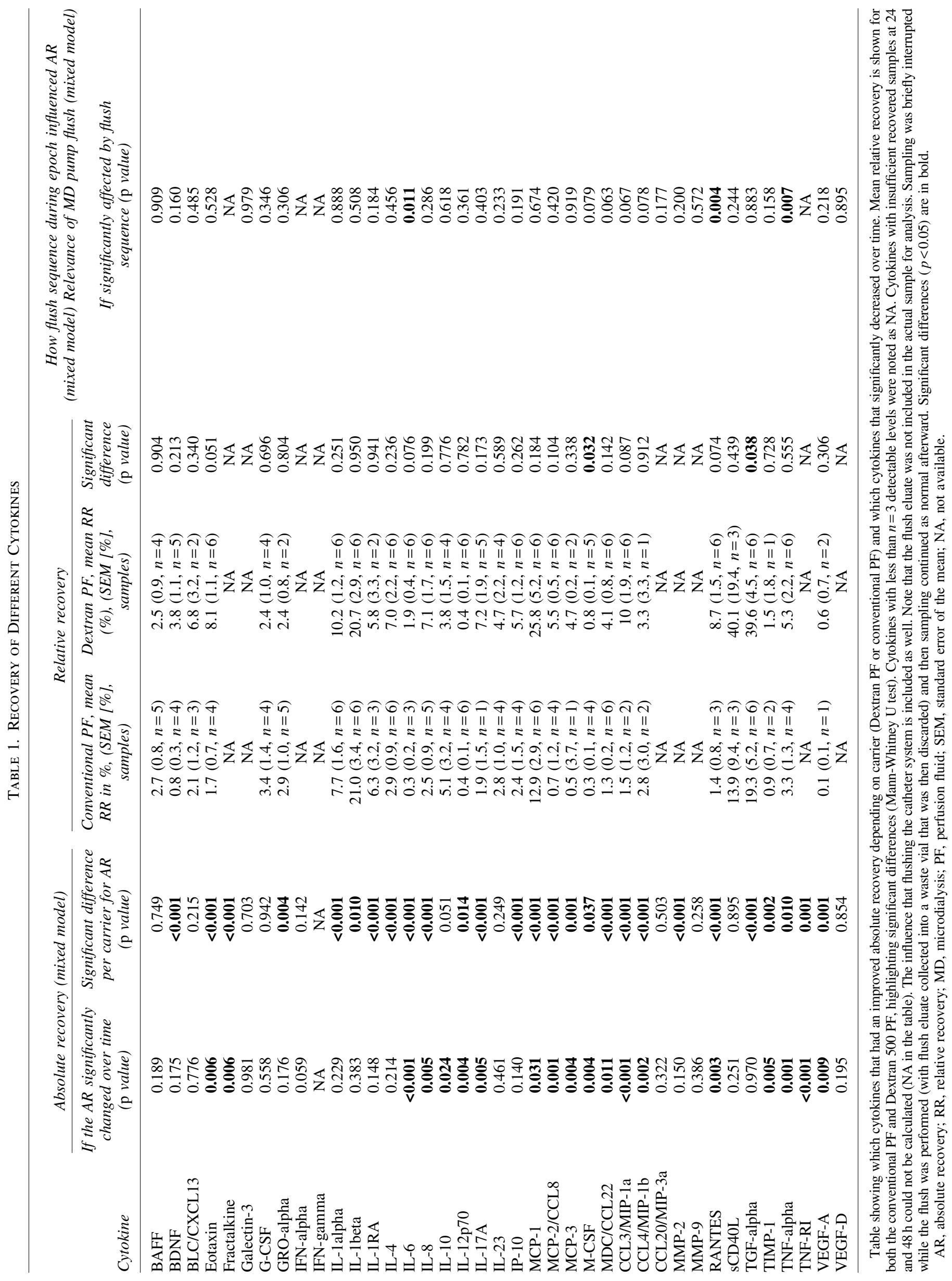


A

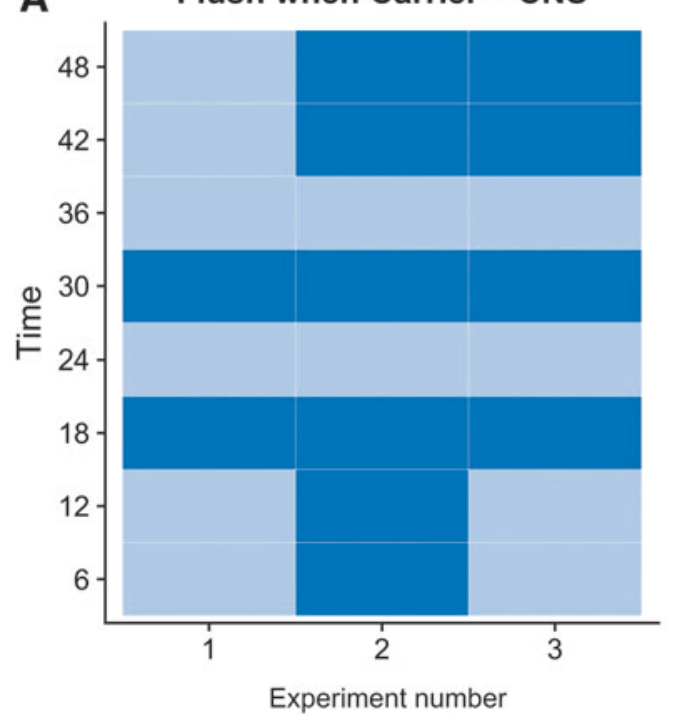

B Flush when Carrier $=$ Dextran

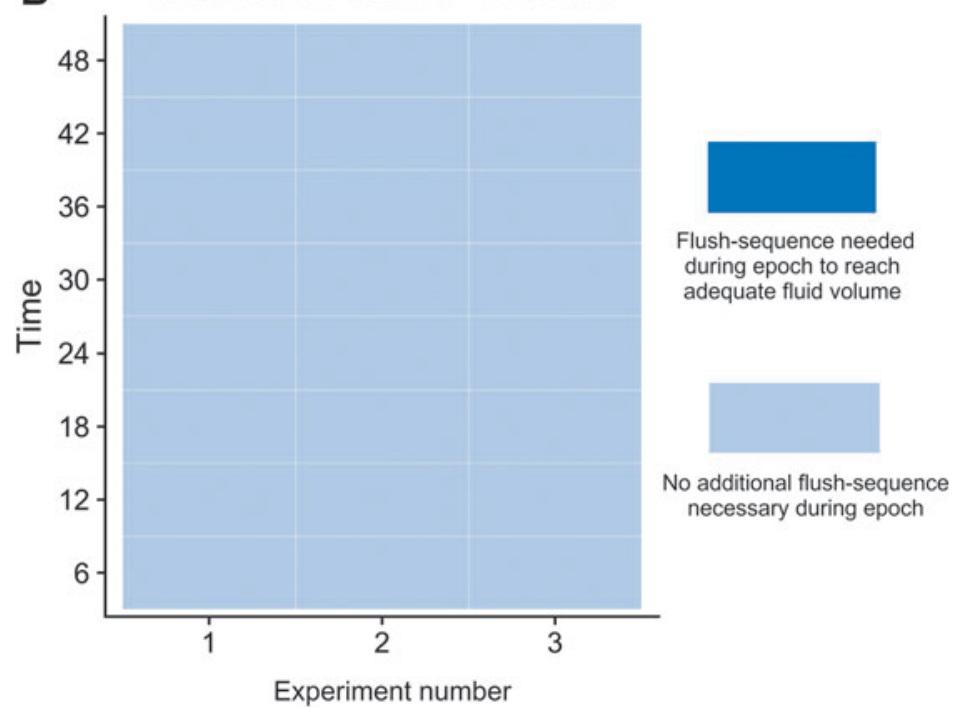

FIG. 2. A time chart of epochs needing MD pump flushing. (A) Catheter perfused with Dextran 500 and (B) conventional (CNS) perfusion fluid. Light blue indicates an adequate fluid recovery whereas dark blue highlights an epoch where a flushing sequence was necessary given that we expected that an inadequate amount would be collected at the end of the epoch. CNS, central nervous system; MD, microdialysis. Color image is available online.

\section{Discussion}

To our knowledge, this is the first study using the CE-marked commercially available Perfusion Fluid CNS Dextran 500 from $\mu$ Dialysis, applicable for human use. This work represents a comprehensive overview of in vitro cytokine/chemokine recovery using this new fluid carrier as compared to the conventional CNS PF. We found that the fluid and absolute recovery were much more robust when the Dextran was used in the carrier fluid. Two cytokines also had a significantly higher RR when Dextran was used in the carrier fluid, suggesting it to be a preferable PF in comparison to conventional CNS PF.

\section{Dextran resulted in an improved fluid recovery}

The catheters perfused with normal CNS PF needed to be "flushed" in $50 \%$ of the epochs to reach expected adequate sample volumes, as compared to the Dextran PF, which always reached
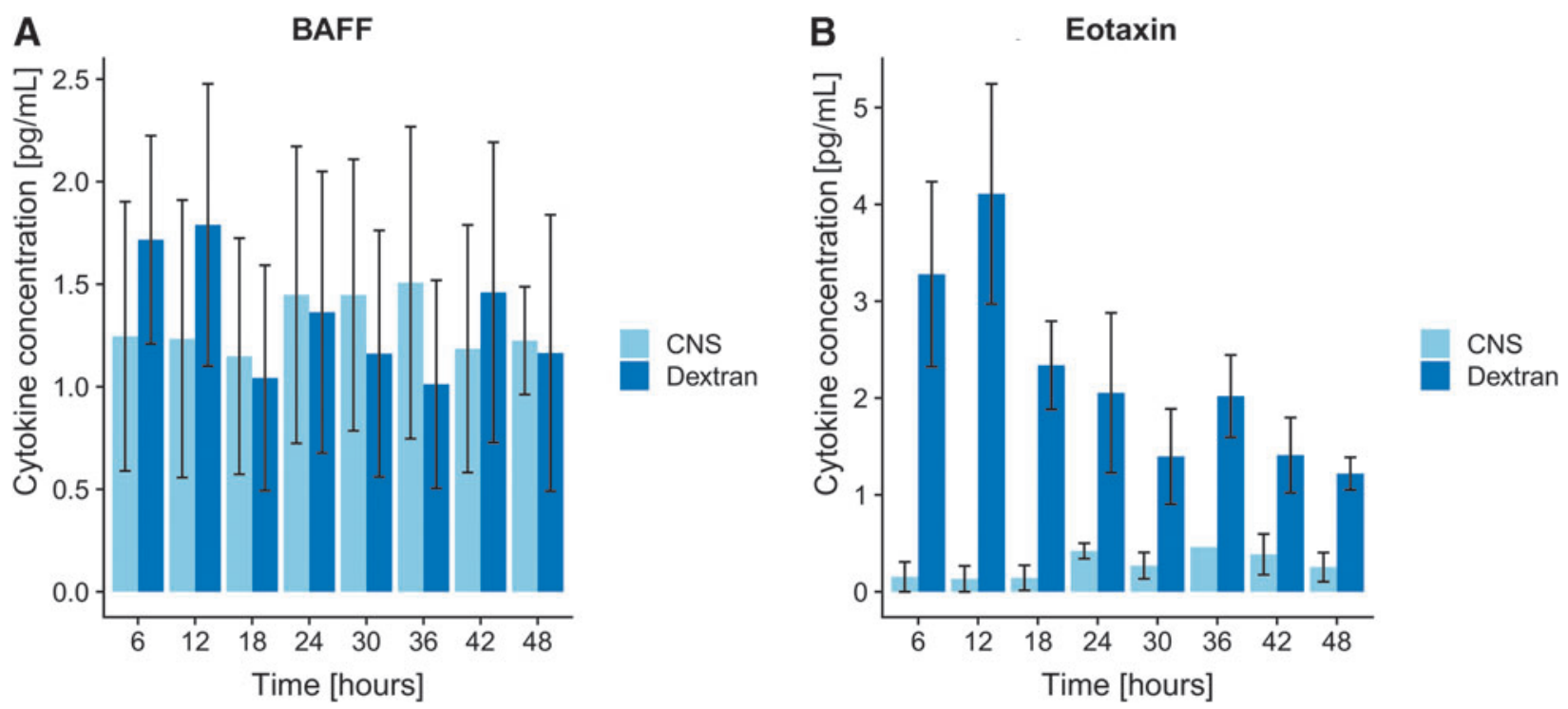

FIG. 3. Examples of absolute recovery over time. (A) An example of a cytokine where the recovery did not differ significantly over time and carrier (BAFF), whereas the recovery of eotaxin (B) did change significantly over time or between the two carriers. The y-axis shows mean cytokine concentration ( $\mathrm{pg} / \mathrm{mL}$ ) with standard error of mean as error bars and $\mathrm{x}$-axis time (hours). BAFF, B-cell activating factor; CNS, central nervous system. Color image is available online. 


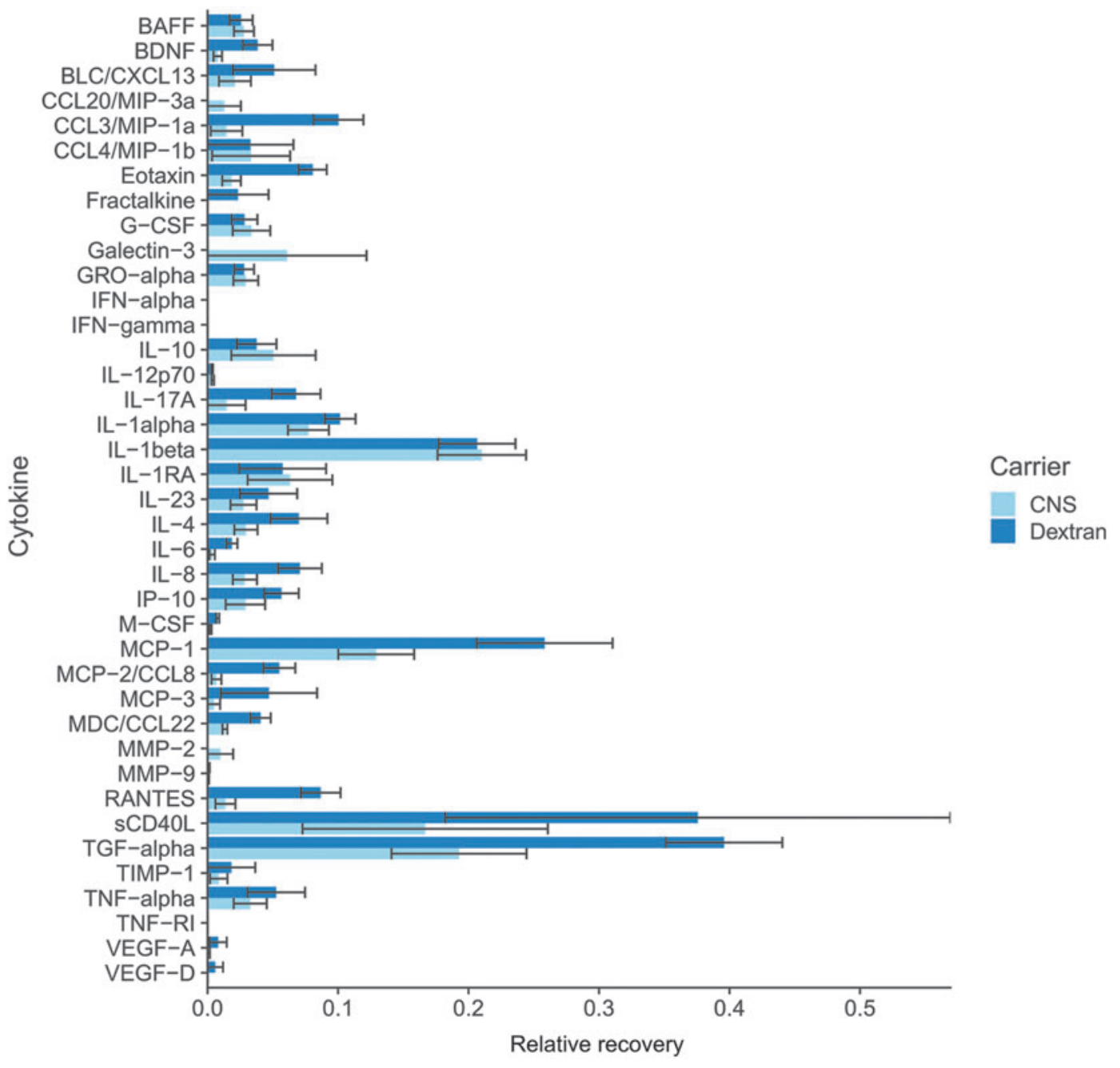

FIG 4. Bar plot of all relative recoveries. Mean relative recoveries (error bars represent standard error of mean) from all 3 independent experiments and pooled 24- and 48-h time points. The y-axis displays the cytokines/chemokines whereas the x-axis shows relative recovery. BAFF, B-cell activating factor; BDNF, brain-derived neurotrophic factor; BLC, B lymphocyte chemoattractant; CNS, central nervous system; CXCL13, chemokine (C-X-C motif) ligand 13; CCL, CC chemokine ligands; G-CSF, granulocyte colony-stimulating factor; GM-CSF, granulocyte-macrophage colony-stimulating factor; GRO, chemokine (C-X-C motif) ligand 1 (CXCL1); IFN, interferon; IL, interleukin; IL-1ra, interleukin-1 receptor antagonist; IP-10/IP10, interferon gamma-induced protein 10 (also known as C-X-C motif chemokine 10 [CXCL10]); MCP-1, monocyte chemotactic protein 1 (also known as CCL2); MCP-3, monocyte chemotactic protein-3 (also known as CCL7); MDC, macrophage-derived chemokine (also known as CCL22); MIP1 $\alpha$, macrophage inflammatory protein 1 alpha (also known as CCL3); MIP1 $\beta$, macrophage inflammatory protein 1 beta (also known as CCL4); PDGF, platelet-derived growth factor; RANTES, regulated on activation, normal T cell expressed and secreted (also known as CCL5); sCD40L, soluble CD40 ligand; sIL-2Ra, soluble interleukin-2 receptor antagonist; TGF, transforming growth factor; TIMP-1, tissue inhibitor of metalloproteinase 1; TNF, tumor necrosis factor; VEGF, vascular endothelial growth factor. Color image is available online.

sufficient volumes of recovery in the collection vials. This is presumably attributed to ultrafiltration causing a fluid loss over the membrane of CNS PF because of low osmotic pressure, ${ }^{9}$ though other explanations, such as varying catheter capabilities depending on the surrounding medium (in vivo vs. in vitro), have also been suggested. ${ }^{6}$ Similarly, in an in vitro recovery study by Dahlin and coworkers, they noticed a $30 \%$ fluid recovery decrease in CNS PF compared to an in-house Dextran 500 solution and only if special surface coated catheters were used (otherwise they did not observe any recovery at all), ${ }^{7}$ similar to Kahl and colleagues using Ringer's solution as PF. ${ }^{16}$ Our $50 \%$ is similar to the previous study from our group comparing $3.5 \%$ albumin with normal CNS PF, which revealed that $44 \%$ of CNS PF epochs had inadequate fluid recovery compared to none using the albumin colloid. ${ }^{6}$ There have been no comparisons between Dextran 500 and 3.5\% albumin PF, but a study from 2005 analyzed Dextran 60 and observed that the fluid recovery was slightly better for albumin, but this difference was insignificant given that both fluid recoveries were almost $100 \% .^{9} \mathrm{It}$ should be noted that the lower fluid volumes we noticed is not as evident for catheters with normal CNS PF placed in brain ECF in vivo, presumably attributed to different pressure gradients and other factors such as endogenous proteins, ${ }^{6,14}$ but a phenomenon more commonly observed in in vitro studies. ${ }^{6,29}$ That being said, we believe the robust fluid recovery observed for Dextran PF is translatable to the clinical scenario akin to the benefit observed with $3.5 \%$ Albumin PF. ${ }^{6}$ In our experience, colloid-supplemented PF 
mitigates against catheter failure during clinical use and reduces the need for catheter replacement. Overall, the Dextran 500 PF shows superior fluid recovery as compared to the normal CNS PF, most likely attributed to the opposing oncotic pressure generated by the colloid within the microdialysis perfusate.

\section{Dextran perfusion fluid improved recovery of cytokine/chemokines}

We found that, overall, the Dextran PF was superior to normal CNS PF in recovery across a range of cytokines. Almost all cytokines had a significantly higher improved AR, and the RRs in the current study were significantly higher for two of the proteins, namely M-CSF and TGF-alpha, using the Dextran PF, compared to CNS PF. Although data on Dextran 500 PF from M Dialysis has never been published before in a similar fashion, Dahlin and coworkers, in Uppsala, Sweden, have escalated Dextran concentrations, and molecular weight of Dextran molecules, and noted an improved recovery of some cytokines for Dextran 500 compared to CNS PF. ${ }^{7}$ However, their study was not structured in a similar fashion as ours, making direct comparisons difficult; but researchers from Uppsala have now shifted to an in-house Dextran 500 as a colloid in PF and successfully recovered larger proteins in swine and rat brain injury models. ${ }^{30,31}$

Our group has previously performed similar in vitro analyses comparing a $3.5 \%$ albumin $\mathrm{PF}$ versus normal $\mathrm{CNS} \mathrm{PF},{ }^{6}$ showing an RR improvement in the colloid in 9 of 12 analyzed cytokines. Further, the RRs in that study reached 30-50\%, often double that of CNS PF. ${ }^{6}$ These RRs were higher than those observed in the current study, which revealed mean RRs of 1-10\%, with some higher responders (e.g., TGF-alpha). We cannot easily explain this apparent disparity with our earlier studies, but this might be partly attributable to the different assay used and more extensive protein-protein interaction attributed to more proteins involved in the present study. In the present study, we used continuous stirring of the external solution. Comparing with the literature, some in vitro recovery studies utilized stirring, whereas some did not. ${ }^{6,32,33}$ It is conceivable that stirring may have an effect on recovery, given that the surface chemistry of interaction with a solution is a potentially complex situation in which layers and gradients can form, potentially affected by stirring. However, these lower RRs seem to be more in line with some previously published studies, ${ }^{32,34-37}$ where RRs in the range of $1-10 \%$ are often noted, although with some reaching higher levels. This distinct heterogeneity in resultsdepending on different study setups-means that caution must be exercised when comparing absolute RR between different studies.

Previous studies have shown that catheters are susceptible to biofouling over time (or even being malformed), decreasing the RR. $^{6,38}$ We compared RRs at 24 versus $48 \mathrm{~h}$, and this was not evident in our data set (data not shown), so even if protein depositions occurred they did not affect the recovery. Instead, a more probable explanation for the decrease in AR is that the ES concentrations decreased for many cytokines over time, which may be attributed to decay of the cytokines in the standard over $48 \mathrm{~h}$ at $37^{\circ} \mathrm{C}$ (Supplementary Table S1. Decreases in RR with time, and in ES concentration with time, in a different in vitro microdialysis setup have previously also been reported, for IL-1alpha, IL-1beta, and interleukin-1 receptor antagonist (IL-1ra). ${ }^{12}$

Dextran 500 and $3.5 \%$ albumin have not been compared directly in a similar fashion. However, Khan and coworkers noted that albumin is preferable to hydroxyethyl starch (HES; similar to dextran) with superior recovery for some cytokines, whereas HES was better for others, tentatively prompting investigators to a choice of PF depending on which cytokine is to be analyzed. ${ }^{16}$

\section{Future aspects and clinical implication for metabolite recovery}

It is unknown what effect (if any) Dextran PF will have on the recovery of clinically analyzed metabolites (e.g., glucose, lactate, and pyruvate) as compared to normal CNS PF. A variant of Dextran 60 in PF was reportedly preferable to saline solutions in PF to recover glucose, ${ }^{13}$ and albumin in $\mathrm{PF}$ has been shown to have lower recovery of lactate compared to other colloids containing different concentrations of HES. ${ }^{16}$ Therefore, Dextran is presumably a preferable choice clinically as compared to albumin and normal CNS PF for the common metabolites, especially if $100-\mathrm{kDa}$ catheters are used. However, before widespread clinical use, moreextensive examinations of RR of normally monitored metabolites need to be performed.

\section{Limitations}

In the context of RR, the study was planned and designed for $n=6$ versus $n=6$ measurements. However, given that many cytokines were not recovered in several epochs, many of these samples returned concentrations below the lower limit of detection by the Luminex assay for this analyte. Although it could be considered a limitation to compare fewer "positive" samples, these "zero" levels presumably represent important information as to highlight which cytokines and concentrations are suitable to recover using microdialysis in the current and similar scenarios. Further, many of the recovered cytokines exhibited a higher variability than expected on the basis of our previous studies. We acknowledge that additional experimental runs would have been desirable in the present study to increase the statistical sample sizes $(n)$, as previously stated. Even so, the current results shed light on the individual cytokines' different propensities for variability in recovery and thus identify those cytokine species that behave most consistently with the current microdialysis technique.

We only compared the effect of the PF in this study, whereas many other factors have been shown to alter microdialysis recovery, including membrane lengths, ${ }^{13}$ membrane coating, ${ }^{31,39}$ fluid pressure, ${ }^{14}$ inclusion of nanoparticles, ${ }^{40}$ and microdialysis pump speed, ${ }^{7}$ which could be other ways to improve the recovery depending on the situation. We have specifically focused on the methodological constraints within clinical practice as a prelude to utilizing this perfusion fluid in clinical studies. As mentioned above, we checked that Dextran PF did not interfere with the Luminex assay. If different analytes are to be measured by other assay techniques, it would be important to perform tests to ascertain whether or not Dextran PF interferes with those assays. For example, preliminary tests we performed with ISCUSflex measurement of glucose, lactate, and pyruvate suggested that Dextran 500 did not interfere with such measurements.

\section{Conclusions}

In vitro studies are not fully representative of the situation in vivo, perhaps attributed to direct tissue/catheter interactions such that the outer boundary of the microdialysis catheter is in contact in vivo with cells, extracellular matrix, and extracellular fluid in the brain tissue, rather than a simple in vitro fluid solution. This makes microdialysis measures such as FR and RR a necessarily crude estimate of the recovery of a given species in vivo. Nevertheless, 
the overall benefit of Dextran PF over conventional PF for the recovery of cytokines and chemokines is supported by these in vitro results, and we therefore regard Dextran $\mathrm{PF}$ as showing promise as a perfusion fluid for use in clinical microdialysis studies requiring recovery of protein.

\section{Acknowledgments}

The work was supported by funding for S.G.C. and K.L.H.C. from the National Institute for Health Research Biomedical Research Centre, Cambridge (Neuroscience Theme; Brain Injury and Repair Theme). P.J.H. is funded by a National Institute for Health Research (NIHR) Professorship, Academy of Medical Sciences/Health Foundation Senior Surgical Scientist Fellowship, and the National Institute for Health Research Biomedical Research Centre, Cambridge. E.P.T. has received salary support from Swedish Society for Medical Research. A.H. is supported by the Royal College of Surgeons of England and the National Institute for Health Research Biomedical Research Centre, Cambridge. The study consumables were purchased through the NIHR Research Professorship (Peter Hutchinson), and the Luminex 200 analyzer was purchased with Medical Research Council (MRC) funding (G0600986 ID79068).

This funding sources had no role in the design of this study, execution, analyses, interpretation of the data, or decision to submit results.

\section{Author Disclosure Statement}

P.J.A.H. is a Director of Technicam, the company that manufactures the triple lumen cranial access device used in the model. P.J.A.H. has no competing non-financial interests with this study. The other authors have no competing financial interests.

\section{References}

1. Thelin, E.P., Carpenter, K.L., Hutchinson, P.J., and Helmy, A. (2017). Microdialysis monitoring in clinical traumatic brain injury and its role in neuroprotective drug development. AAPS J. 19, 367-376.

2. Hutchinson, P.J., Jalloh, I., Helmy, A., Carpenter, K.L., Rostami, E., Bellander, B.M., Boutelle, M.G., Chen, J.W., Claassen, J., DahyotFizelier, C., Enblad, P., Gallagher, C.N., Helbok, R., Hillered, L., Le Roux, P.D., Magnoni, S., Mangat, H.S., Menon, D.K., Nordstrom, C.H., O'Phelan, K.H., Oddo, M., Perez Barcena, J., Robertson, C., Ronne-Engstrom, E., Sahuquillo, J., Smith, M., Stocchetti, N., Belli, A., Carpenter, T.A., Coles, J.P., Czosnyka, M., Dizdar, N., Goodman, J.C., Gupta, A.K., Nielsen, T.H., Marklund, N., Montcriol, A., O’Connell, M.T., Poca, M.A., Sarrafzadeh, A., Shannon, R.J., SkjothRasmussen, J., Smielewski, P., Stover, J.F., Timofeev, I., Vespa, P., Zavala, E,. and Ungerstedt, U. (2015). Consensus statement from the 2014 International Microdialysis Forum. Intensive Care Med. 41, 1517-1528.

3. Duo, J., Fletcher, H., and Stenken, J.A. (2006). Natural and synthetic affinity agents as microdialysis sampling mass transport enhancers: current progress and future perspectives. Biosens. Bioelectron. 22, 449-457.

4. Benveniste, H., and Huttemeier, P.C. (1990). Microdialysis-theory and application. Prog. Neurobiol. 35, 195-215.

5. Hillered, L., Dahlin, A.P., Clausen, F., Chu, J., Bergquist, J., Hjort, K., Enblad, P., and Lewen, A. (2014). Cerebral microdialysis for protein biomarker monitoring in the neurointensive care setting - a technical approach. Front. Neurol. 5, 245.

6. Helmy, A., Carpenter, K.L., Skepper, J.N., Kirkpatrick, P.J., Pickard, J.D., and Hutchinson, P.J. (2009). Microdialysis of cytokines: methodological considerations, scanning electron microscopy, and determination of relative recovery. J. Neurotrauma 26, 549-561.

7. Dahlin, A.P., Wetterhall, M., Caldwell, K.D., Larsson, A., Bergquist, J., Hillered, L., and Hjort, K. (2010). Methodological aspects on mi- crodialysis protein sampling and quantification in biological fluids: an in vitro study on human ventricular CSF. Anal. Chem. 82, 4376-4385.

8. Keeler, G.D., Durdik, J.M., and Stenken, J.A. (2014). Comparison of microdialysis sampling perfusion fluid components on the foreign body reaction in rat subcutaneous tissue. Eur. J. Pharm. Sci. 57, 60-67.

9. Hillman, J., Aneman, O., Anderson, C., Sjogren, F., Saberg, C., and Mellergard, P. (2005). A microdialysis technique for routine measurement of macromolecules in the injured human brain. Neurosurgery $56,1264-1268$; discussion, 1268-1270.

10. Helmy, A., Carpenter, K.L., Menon, D.K., Pickard, J.D., and Hutchinson, P.J. (2011). The cytokine response to human traumatic brain injury: temporal profiles and evidence for cerebral parenchymal production. J. Cereb. Blood Flow Metab. 31, 658-670.

11. Helmy, A., De Simoni, M.G., Guilfoyle, M.R., Carpenter, K.L., and Hutchinson, P.J. (2011). Cytokines and innate inflammation in the pathogenesis of human traumatic brain injury. Prog. Neurobiol. 95, 352372 .

12. Hutchinson, P.J., O'Connell, M.T., Rothwell, N.J., Hopkins, S.J., Nortje, J., Carpenter, K.L., Timofeev, I., Al-Rawi, P.G., Menon, D.K., and Pickard, J.D. (2007). Inflammation in human brain injury: intracerebral concentrations of IL-1alpha, IL-1beta, and their endogenous inhibitor IL-1ra. J. Neurotrauma 24, 1545-1557.

13. Hamrin, K., Rosdahl, H., Ungerstedt, U., and Henriksson, J. (2002). Microdialysis in human skeletal muscle: effects of adding a colloid to the perfusate. J. Appl. Physiol. 92, 385-393.

14. Chu, J., Hjort, K., Larsson, A., and Dahlin, A.P. (2014). Impact of static pressure on transmembrane fluid exchange in high molecular weight cut off microdialysis. Biomed. Microdevices 16, 301-310.

15. Schutte, R.J., Oshodi, S.A., and Reichert, W.M. (2004). In vitro characterization of microdialysis sampling of macromolecules. Anal. Chem. 76, 6058-6063.

16. Khan, F., Pharo, A., Lindstad, J.K., Mollnes, T.E., Tonnessen, T.I., and Pischke, S.E. (2015). Effect of perfusion fluids on recovery of inflammatory mediators in microdialysis. Scand. J. Immunol. 82, 467-475.

17. Mellergard, P., Sjogren, F., and Hillman, J. (2010). Release of VEGF and FGF in the extracellular space following severe subarachnoidal haemorrhage or traumatic head injury in humans. Br. J. Neurosurg. 24, 261-267.

18. Chu, J., Koudriavtsev, V., Hjort, K., and Dahlin, A.P. (2014). Fluorescence imaging of macromolecule transport in high molecular weight cut-off microdialysis. Anal. Bioanal. Chem. 406, 7601-7609.

19. R Core Team. (2018). R: A language and environment for statistical computing. R Foundation for Statistical Computing: Vienna, Austria.

20. Wickham, H. (2017). tidyverse: Easily Install and Load the 'Tidyverse'. https://cran.r-project.org/package=tidyverse

21. O. Wilke, C. (2018). cowplot: Streamlined Plot Theme and Plot Annotations for 'ggplot2'.

22. Neuwirth, E. (2014). RColorBrewer: ColorBrewer Palettes. https:// cran.r-project.org/package=RColorBrewer

23. Pinheiro, J., Bates, D., DebRoy, S., Sarkar, D., and R Core Team. (2018). nlme: Linear and Nonlinear Mixed Effects Models. https:// cran.r-project.org/package $=$ nlme

24. West, B., Welch, K., and Galecki, A. (2015). Linear Mixed Models: A Practical Guide Using Statistical Software, 2nd ed. Chapman and Hall/CRC: Croydon, UK.

25. Bates, D., Maechler, M., Bolker, B. and Walker, S. (2015). Fitting linear mixed-effects models using lme4. J. Stat. Softw. 67, 1-48.

26. Kuznetsova, A., Brockhoff, P.B., and Christensen, R.H.B. (2017). lmerTest Package: tests in linear mixed effects models. J. Stat. Softw. $82,1-26$.

27. Robinson, D., and Hayes, A. (2018). broom: convert statistical analysis objects into tidy tibbles. https://cran.r-project.org/package=broom

28. Sarkar, D. (2008). Lattice: Multivariate Data Visualization with $R$. Springer: New York.

29. Chefer, V.I., Thompson, A.C., Zapata, A., and Shippenberg, T.S. (2009). Overview of brain microdialysis. Curr. Protoc. Neurosci. Chapter 7, Unit7.1.

30. Dahlin, A.P., Purins, K., Clausen, F., Chu, J., Sedigh, A., Lorant, T., Enblad, P., Lewen, A., and Hillered, L. (2014). Refined microdialysis method for protein biomarker sampling in acute brain injury in the neurointensive care setting. Anal. Chem. 86, 8671-8679.

31. Clausen, F., Marklund, N., and Hillered, L. (2019). Acute inflammatory biomarker responses to diffuse traumatic brain injury in the rat monitored by a novel microdialysis technique. J Neurotrauma 36, 201-211. 
32. Ao, X., and Stenken, J.A. (2006). Microdialysis sampling of cytokines. Methods 38, 331-341.

33. Marcus, H.J., Carpenter, K.L., Price, S.J., and Hutchinson, P.J. (2010). In vivo assessment of high-grade glioma biochemistry using microdialysis: a study of energy-related molecules, growth factors and cytokines. J. Neurooncol. 97, 11-23.

34. Ao, X., Sellati, T.J., and Stenken, J.A. (2004). Enhanced microdialysis relative recovery of inflammatory cytokines using antibody-coated microspheres analyzed by flow cytometry. Anal. Chem. 76, 37773784.

35. Sjogren, F., Svensson, C., and Anderson, C. (2002). Technical prerequisites for in vivo microdialysis determination of interleukin-6 in human dermis. Br. J. Dermatol. 146, 375-382.

36. Trickler, W.J., and Miller, D.W. (2003). Use of osmotic agents in microdialysis studies to improve the recovery of macromolecules. J. Pharm. Sci. 92, 1419-1427.

37. Waelgaard, L., Pharo, A., Tonnessen, T.I., and Mollnes, T.E. (2006). Microdialysis for monitoring inflammation: efficient recovery of cytokines and anaphylotoxins provided optimal catheter pore size and fluid velocity conditions. Scand. J. Immunol. 64, 345-352.

38. Wisniewski, N., Klitzman, B., Miller, B., and Reichert, W.M. (2001). Decreased analyte transport through implanted membranes: differen- tiation of biofouling from tissue effects. J. Biomed. Mater. Res. 57, 513-521.

39. Dahlin, A.P., Hjort, K., Hillered, L., Sjodin, M.O., Bergquist, J., and Wetterhall, M. (2012). Multiplexed quantification of proteins adsorbed to surface-modified and non-modified microdialysis membranes. Anal. Bioanal. Chem. 402, 2057-2067.

40. Giorgi-Coll, S., Blunt-Foley, H., Hutchinson, P.J., and Carpenter, K.L.H. (2017). Heparin-gold nanoparticles for enhanced microdialysis sampling. Anal. Bioanal. Chem. 409, 5031-5042.

Address correspondence to:

Eric Peter Thelin, MD, PhD Karolinska Universitetssjukhuset Solna

BioClinicum J5:20

Tema Neuro

forskargrupp Svensson S-171 76 Stockholm Sweden

E-mail: eric.thelin@ki.se 